\title{
Chapter 14 \\ Detection of Damage in Metallic Structures for Offshore Applications
}

\author{
Rohan Soman, Paweł Malinowski, and Wiesław Ostachowicz
}

\begin{abstract}
Wind energy is seen as one of the most promising solutions to man's ever increasing demands of a clean source of energy. However, a major drawback of the wind energy is the high initial cost for setting up and maintenance. This makes the energy more expensive than the conventional energy sources like fossil fuels and nuclear and hence it has not been widely accepted. Thus, there is a concerted effort to reduce the cost of energy production. This can be achieved by increasing the lifetime of the wind turbines; reducing maintenance costs and ensuring low downtime of the turbine. The lifetime may be increased by ensuring a more robust design while the maintenance cost and the downtime of the equipment may be lowered through the use of condition monitoring (CM) and structural health monitoring (SHM). SHM allows early detection of damage and allows maintenance planning which becomes more important in the off-shore industry where the cost of unscheduled maintenance are high due to the need of specialized equipment. Also, maintenance planning can allow us to avoid unnecessary downtime, hence increasing the availability of the system. In wind turbine structures, tower damage is the third most common damage in wind turbines. Thus, this work concentrates on SHM of a wind turbine tower. A decision level data fusion based on bi-axial tracking of change in Neutral Axis (NA) position is proposed. A discrete Kalman Filter (KF) is employed for the estimation of the NA in the presence of measurement noise from the strain sensors. The KF allows data fusion from the strain sensors and the yaw mechanism for the accurate estimation of the NA. Any change in the NA position may be used as an indicator for the presence and location of the damage. The ratio of the change in the NA along two perpendicular axes is taken and used for the localization. The study has been carried out on the simulated finite element (FE) model of the wind turbine tower and indicates that bi-axial NA tracking based on data fusion is indeed necessary and at the same time is sensitive to damage. The proposed methodology is then validated on real strain data from the Nordtank NTK 500/41 wind turbine. Based on the results presented, the change in NA is indeed a robust damage indicator insensitive to ambient condition changes, and the applied loads.
\end{abstract}

R. Soman $(\bowtie) \bullet$ P. Malinowski $\bullet$ W. Ostachowicz

Mechanics of Intelligent Structures Department, Institute of Fluid Flow Machinery, Polish

Academy of Sciences, ul. Fiszera 14, 80-231 Gdańsk, Poland

e-mail: rsoman@imp.gda.pl; pawel.malinowski@imp.gda.pl; wieslaw@imp.gda.pl

(C) The Author(s) 2016

W. Ostachowicz et al. (eds.), MARE-WINT, DOI 10.1007/978-3-319-39095-6_14 


\subsection{Introduction}

The main hindrance for more widespread deployment of the wind turbines is the high initial cost for setting up of wind farms and its subsequent maintenance. These high initial costs make the energy more expensive than the conventional energy sources. The cost of generation being the biggest drawback of wind energy, there is a concerted effort to reduce it. This can be achieved by increasing the life-time of the wind turbines; reducing maintenance costs and ensuring high availability. The lifetime may be increased by ensuring a more robust design while the maintenance cost may be lowered and the high availability ensured through the use of condition monitoring (CM) and structural health monitoring (SHM) (Cho et al. 2010). SHM allows early detection of damage and allows maintenance planning which reduces the cost (Doebling et al. 1998). Furthermore, it can allow us to avoid unnecessary downtime, hence increasing the availability of the system.

The SHM needs to be low cost, and suitable for continuous monitoring. These techniques are based on the concept that, the change in mechanical properties of the structure will be captured by a change in its dynamic characteristics (Adewuyi et al. 2009). The SHM process involves the observation of a system over time using periodically sampled dynamic response measurements from an array of sensors, followed by the extraction of damage-sensitive features from these measurements, and the statistical analysis of these features to determine the current state of the system's health. The SHM process requires use of sensors for data collection, filters for data cleansing, and central data processing units for feature extraction and post processing.

SHM has been a field of active research in the aerospace and offshore industry for several decades and many methods have been proposed and investigated. These methods have been able to detect and locate damage in a laboratory environment under controlled conditions. However when these methods were implemented for field validations or in real structures, the results obtained were not up to the desired level. The discrepancy between the expected and the measured results are mainly attributed to the uncertainty in the measurement environment with respect to noise, temperature and excitation mechanism for the structure. Hence there is a search for an SHM system which is able to detect damage in working conditions, and is robust enough to changes in ambient conditions and excitation. Furthermore, the method should be able to detect small levels of damage.

The lack of a standard method for designing an SHM system which will enable us to perform accurate damage detection is the primary motivation of this research.

\subsection{Methodology Overview}

The scope of the research is SHM of Tower Structures through the measurement of the strain in the tower subject to in-service loading. The methodology is explained here. 


\subsubsection{Neutral Axis}

The primary function of the tower structure is to support the hub, and the nacelle of the wind turbine. The nacelle and the hub are axial loads which are eccentrically loaded on the tower. This eccentric loading gives rise to axial compressive loads as well as bending loads as shown in Fig. 14.1. The axial compression is uniform over the entire cross section while the bending loads will be tensile at one end and compressive at the other. Furthermore, the tower experiences wind loads which result in bending strains in the tower. The axial strains are given by Eq. (14.1):

$$
\varepsilon_{\text {axial }}=\frac{F}{E A}
$$

where, $\varepsilon_{\text {axial }}$ is the longitudinal strain in bending, $F$ is the net axial force due to the nacelle, hub and other wind turbine components, $E$ is the Young's modulus and $A$ is the area of the cross section. Equation (14.2) gives the bending

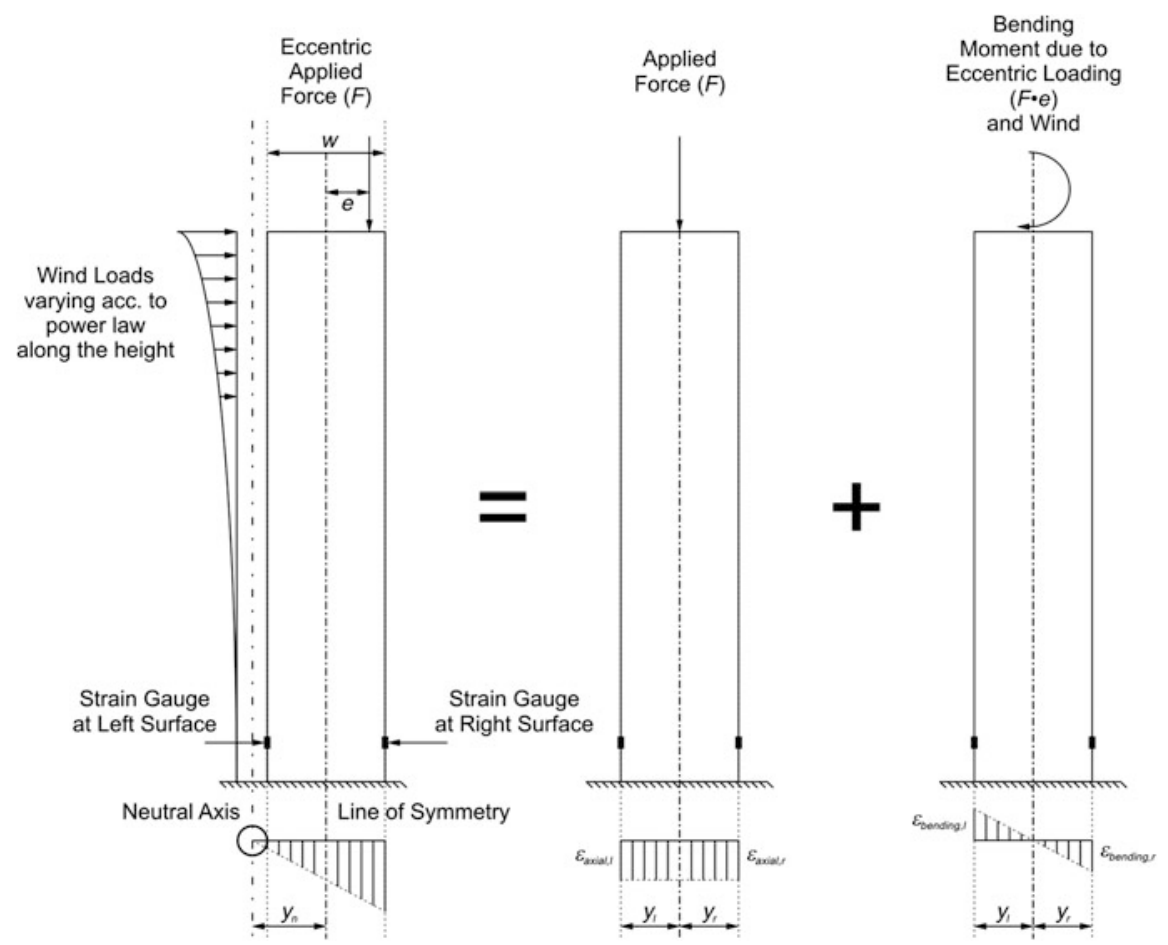

Fig. 14.1 Flexural strain distribution over the cross-section subject to eccentric loading (Soman et al. 2014b) 
strains:

$$
\varepsilon_{\text {bending }}=\frac{M_{b} y}{E I}
$$

where, $\varepsilon_{\text {bending }}$ is the longitudinal strain in bending, $M_{b}$ is the net bending moment at the cross section due to wind loading and eccentricity, and $I$ is the area moment of inertia, $y$ is the distance from the NA to the location of the sensor (Xia et al. 2012).

Thus, one surface of the tower experiences, a combination of two axial compressions, (right side, Fig. 14.1) while the other end experiences a combination of compressive load due to the weight and tensile load due to the bending (left side, Fig. 14.1). The net strains on the right and left surface in Fig. 14.1 are given by Eq. (14.3) (Soman et al. 2015b):

$$
\varepsilon_{r / l}=-\varepsilon_{\text {axial }} \pm \varepsilon_{\text {bending }}
$$

If the line connecting the two strain levels is extended, there will be a point where the strain experienced will be zero, which is identified as the NA point. The NA of the section is a function of the flexural rigidity of the structure, and does not depend on the applied bending loads, thus by, measuring the strains at the opposite edges of the beam, the NA can be located, which in turn may be used as an indicator of the damage. Figure 14.1 explains the abbreviations used and the concept. The NA can thus be estimated based on the strain measurements. This point may be found out by linear extrapolation, and is given by Eq. (14.4) (Soman et al. 2015b):

$$
L=\frac{\left(\varepsilon_{\text {bending }}-\varepsilon_{\text {axial }}\right) \cdot w}{2 \cdot \varepsilon_{\text {bending }}}=\frac{\varepsilon_{l} \cdot w}{\left(\varepsilon_{l}-\varepsilon_{r}\right)}
$$

where, L is the NA Location. It should be noted that the dimensions of the NA location are that of distance, so this metric may be represented as a dimensionless quantity given by Eq. (14.5) (Soman et al. 2015b):

$$
N A E=\frac{L}{w}
$$

The NAE can be made at each time instant based on measured strains at that instance using Eq. (14.5). This approach is termed as direct estimation in further sections, or they can be estimated based on the previous estimates and updated at each time instant using the new measurements using KF which is explained in detail in the later section. 


\subsubsection{Damage Sensitive Feature}

As mentioned, in earlier section, the NA location is independent of the loading conditions, and depends only on the condition of the structure. Thus the $\%$ change in the NAE is taken as the damage sensitive feature and is given by Eq. (14.6) (Soman et al. 2015a):

$$
\triangle N A E=\frac{N A E_{\text {healthy }}-N A E_{\text {monitored }}}{N A E_{\text {healthy }}} \times 100 \%
$$

The NAE $\mathrm{E}_{\text {healthy }}$ is developed at the time of the installation of sensors when the structure is known to be in healthy condition. $\mathrm{NAE}_{\text {monitored }}$ is the estimate at every time step. If the \% change exceeds a certain threshold, an alarm is raised indicating damage. This threshold is based on sensitivity studies and the tradeoff between the probability of false positive and false negative detections.

\subsubsection{Bi-Axial NA tracking}

The aim of the SHM system is accurate Level II damage detection. In order to locate the damage, one needs the coordinates of the damage location along the $\mathrm{x}$; $\mathrm{y} ; \mathrm{z}$ axes. The sensor placement at diametrically opposite locations along the tower, as shown in Fig. 14.1, if extended by placing an array of sensors in a line along the tower, can locate the damage along the altitude ( $\mathrm{z}$ axis). In order to detect the location along the circumference of the tower, we need to perform bi-axial neutral axis tracking. The sensor pairs are placed along mutually perpendicular axes to map the $\mathrm{x}$ and $\mathrm{y}$ coordinates of damage location. In the polar coordinates, this location can be detected by the angle alone. By the mutually perpendicular sensor placement, the damage can be observed in the form of the sine and the cosine component. The change along $\mathrm{x}$ axis is given by the cosine component while along the $\mathrm{y}$ axis is given by the sine component of the angle. Thus knowing these components one can determine by taking the tan inverse of the ratio of the changes in the sine and cosine as shown in Eq. (14.7) (Soman et al. 2015b):

$$
D L=\tan ^{-1}\left(\frac{\Delta N A E_{\text {yaxis }}}{\Delta N A E_{\text {xaxis }}}\right)
$$

where $D L$ is the damage location in terms of angle $(\phi)$.

It should be kept in mind that the periodicity for sine and cosine is $2 \pi$ while that for tan ratio is $\pi$ thus leading to loss of directionality. Thus a decision level data fusion is necessary, where the change in the directionality is overcome by proper study of the change in the NA location along both the sensor axes. The sign of the change in the NAE of both the axes determine the exact location of the damage. 


\subsubsection{Kalman Filter}

The KF is a set of mathematical equations that provides an efficient computational (recursive) solution of the least squares method (Welch and Bishop 1995). Theoretically, KF combines a system's dynamic model (physical laws of motion) and measurements (sensor readings) to form an estimate of the systems varying quantities (system state) that is better than the estimate of the system obtained by measurement alone (Welch and Bishop 1995).

The KF allows estimation of the state variable $\boldsymbol{x}$ of a discrete time controlled process governed by the linear stochastic difference equation. In the absence of the control scalar, it can be given by Eq. (14.8) (Brown and Hwang 1997):

$$
\boldsymbol{x}_{k+1}=\boldsymbol{A}_{k} \boldsymbol{x}_{k}+\boldsymbol{w}_{k}
$$

where $\boldsymbol{A}$ is the state transition matrix and $\boldsymbol{w}_{k}$ is the white process noise, $k$ indicates the time step. The measurement equation is given by Eq. (14.9):

$$
\boldsymbol{z}_{k}=\boldsymbol{H} \boldsymbol{x}_{k}+\boldsymbol{v}_{k}
$$

where $\boldsymbol{H}$ is the measurement matrix that relates to the observation $\boldsymbol{z}_{k}$ and $\boldsymbol{v}_{k}$ is the measurement noise.

The goal of using KF is to find an equation that computes a posteriori state $\mathrm{x}_{k / k}$ as a linear combination of an a priori estimate $\boldsymbol{x}_{k / k-1}$ and a weighted difference between an actual measurement $\boldsymbol{z}_{k}$ and a measurement prediction $\boldsymbol{H} \boldsymbol{x}_{\mathrm{k} / \mathrm{k}-1}$ as shown in Eq. (14.10):

$$
\boldsymbol{x}_{k / k}=\boldsymbol{x}_{k / k-1}+\boldsymbol{K}\left(\boldsymbol{z}_{k}-\boldsymbol{H} \boldsymbol{x}_{k / k-1}\right)
$$

where $\boldsymbol{K}$ is the Kalman gain. The value of $\boldsymbol{K}$ is chosen to minimise the error covariance. There are several forms of the $\boldsymbol{K}$ matrix discussed in literature. One form of this matrix used for the gain computation is given by Eq. (14.11):

$$
\boldsymbol{K}_{k}=\boldsymbol{P}_{k / k-1} \boldsymbol{H}_{k}^{T}\left(\boldsymbol{H}_{k} \boldsymbol{P}_{k / k-1} \boldsymbol{H}_{k}^{T}+\boldsymbol{R}_{k}\right)^{-1}
$$

where, $\boldsymbol{P}$ is the error covariance matrix, and $\boldsymbol{R}_{k}$ is the measurement noise covariance matrix.

Figure 14.2 concisely explains the implementation of the KF in the present application.

The state estimate variable is $\boldsymbol{X}_{k}=[N A E, 1, \theta]^{T}$, NAE in undamaged condition should remain constant independent of the applied loads, the second variable tracked is the constant value 1 . This constant is incorporated to ensure, correct relation between the state estimation matrix, the observation matrix and the measurement matrix. The added benefit of the constant is, it makes the measurement matrix square, which allows faster computations. The third component of the vector is the 


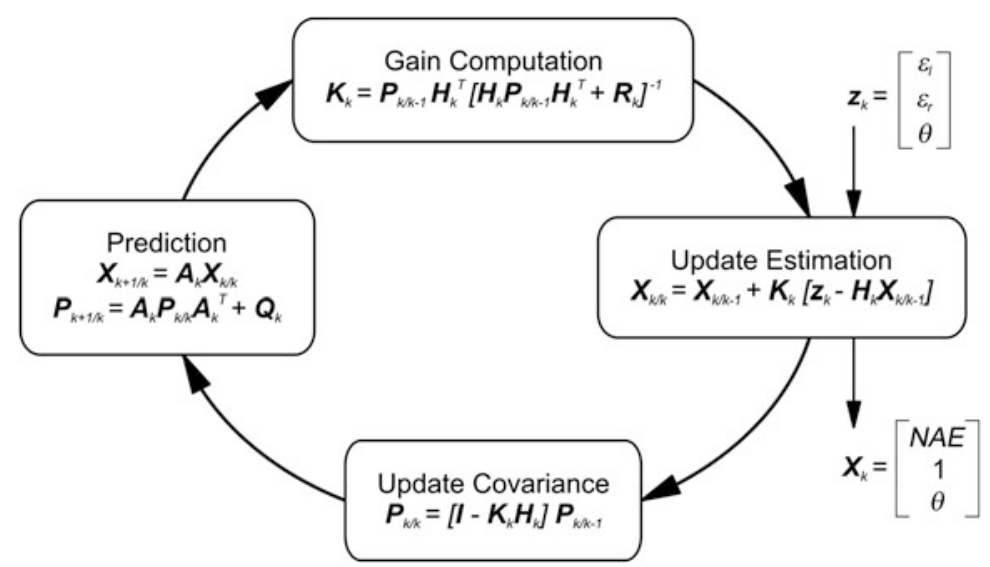

Fig. 14.2 Flow chart for the implementation of the KF (Soman et al. 2014a)

variable $\theta$ for the yaw angle. It is a linear estimate of the measurement from the sensor. The input for the KF algorithm is essentially the state transition matrix $(\boldsymbol{A})$, which relates the state estimate variable in time. In this case, $\boldsymbol{A}$ is a unity matrix of dimension 3 as the state estimates are random and not co-related in time. The other input is the measurement matrix $(\boldsymbol{H})$ which links the state estimation variable $\boldsymbol{X}_{k}$, and the measurement variable $\left(\boldsymbol{Z}_{\boldsymbol{k}}\right)$ at each time step (measurement from the sensors).

The $\mathrm{H}$ matrix takes into consideration the observability of the NA based on the locations of the sensors and is designed for accurate system modelling while maintaining the linearity of the measurement step. In the present case, $\boldsymbol{Z}_{\boldsymbol{k}}=\left[\varepsilon_{l}, \varepsilon_{r}\right.$, $\theta]^{T}$ vector consisting of the strain measurements from the left $\left(\varepsilon_{l}\right)$ and the right side $\left(\varepsilon_{r}\right)$ of the tower and the yaw angle measurement $(\theta)$ of the nacelle.

\subsection{Structure Modelling}

The mathematical formulation and the theory discussed in Sect. 14.2 was applied on a FE model of the DTU $10 \mathrm{MW}$ Reference Wind Turbine (RWT) (Bak et al. 2013 ) for numerical validation. The theory was later validated on the real strain data from the Nordtank NTK 500/41 wind turbine (Paulsen 2011). This section gives the overview of the structures used for the validation and the details of the FE modelling of these structures. 


\subsubsection{DTU $10 \mathrm{MW} R \mathrm{RT}$}

The tower was modelled using the geometric and material properties from the design drawings in commercial FE software ABAQUS. The tower structure was modelled with S4R shell elements. For studying the behavior of the tower to wind loading the dynamic direct analysis was to be carried out. The dynamic analysis uses the modal superposition for estimating the displacements and the strain, so in order to achieve accurate results, and limit the computational load the number of extracted mode shapes was 50. The mesh size of the element was then chosen in order to achieve stable and smooth mode shapes for the extracted modes. The tower was divided in to 82 elements along the height and 48 elements around the circumference. This allowed a reasonable aspect ratio of the shell elements, which lead to a more stable and reliable computation. The tower was encased at the bottom (all DOFs at bottom fixed). The nacelle and hub loads were applied as point loads, at specified eccentricity and height indicated from the design specifications. The wind loads were simulated as random loads using Euro-codes (Standards Norway 2009). A peak wind pressure was selected and applied on the surface area facing the wind, in order to compute the force. The force increases according to the power law along the height of the tower (Sen et al. 2012). The blades, however, were assumed to be pitched into a full aerodynamic brake position to ensure minimal rotor motion and consequent change in mass distribution, which may affect the NA (Bas et al. 2012).

\subsubsection{Nordtank NTK 500/41 Wind Turbine}

The methodology proposed in Sect. 14.2 was validated from real strain data from the Nordtank wind turbine. The test wind turbine, is located at Ris $\emptyset$ Campus, Roskilde, and is a traditional Danish three-bladed stall regulated Nordtank (see specifications in Table 14.1).

The wind turbine is instrumented with a variety of sensors on the blades, in the gearbox and on the tower for measuring the different response of the structure and the ambient conditions like strain, the acceleration response to wind loading, the wind speed and direction, the yaw angle, temperature etc. Some of these sensors, namely the strain sensors at the bottom of the tower, the wind speed and direction sensors and the yaw angle measurements have been used for the validation of the methodology. The type of the sensor and its description is given in Table 14.2 
Table 14.1 Nordtank NTK 500/41 specifications (Paulsen 2011)

\begin{tabular}{l|l}
\hline \multicolumn{2}{l}{ Wind turbine } \\
\hline 8 Risø-R-1593(EN) & \\
\hline Mechanical brake & High speed shaft, operated in FS-mode \\
\hline Power regulation & Passive aerodynamic stall \\
\hline Gearbox & Flender; ratio 1:55.35 \\
\hline Generator & Siemens $500 \mathrm{~kW}, 4$ poles, 690 V \\
\hline Blade profile & NACA 63-4xx \& NACA FF-W3, equipped with vortex generators \\
\hline Tower type & $33.8 \mathrm{~m}$ \\
\hline Conical steel tube & $36.0 \mathrm{~m}$ \\
\hline Hub height & $1960 \mathrm{~kg}$ \\
\hline Masses & $9030 \mathrm{~kg}$ \\
\hline Blade weight & $24,430 \mathrm{~kg}$ \\
\hline Rotor including hub & $22,500 \mathrm{~kg}$ \\
\hline Tower head mass &
\end{tabular}

Table 14.2 Relevant sensor specifications (Paulsen 2011)

\begin{tabular}{l|l|l|l|l}
\hline Measurement & Sensor & Signal type & $\begin{array}{l}\text { Conversion } \\
\text { principle }\end{array}$ & Transmitter \\
\hline $\begin{array}{l}\text { Wind speed } \\
\text { nacelle }\end{array}$ & $\begin{array}{l}\text { Risø cup } \\
\text { anemometer } \\
\text { P2546A with } \\
\text { Reed relay }\end{array}$ & Digital & $\begin{array}{l}\text { 2 magnets on } \\
\text { turning shaft } \\
\text { controls the } \\
\text { contact closure } \\
\text { timings of the } \\
\text { relay per } \\
\text { revolution }\end{array}$ & $\begin{array}{l}\text { Risø P2858A } \\
\text { DAU configured } \\
\text { to periodic time } \\
\text { measurement }\end{array}$ \\
\hline $\begin{array}{l}\text { Wind direction } \\
\text { nacelle }\end{array}$ & $\begin{array}{l}\text { Vector Wind vane } \\
\text { F2819A }\end{array}$ & Analogue & $\begin{array}{l}\text { Measuring on } \\
\text { resistive viper } \\
\text { path relative to } \\
\text { one full turn }\end{array}$ & Voltage divider \\
\hline $\begin{array}{l}\text { Tower bottom } \\
\text { bending moment } \\
\text { X }\end{array}$ & $\begin{array}{l}\text { Micro } \\
\text { Measurement } \\
\text { Strain Gauge } \\
\text { CEA-06-250C- } \\
\text { 350 }\end{array}$ & Analogue & $\begin{array}{l}\text { Semiconductor } \\
\text { foil subjected to } \\
\text { resistive change } \\
\text { due to bending }\end{array}$ & $\begin{array}{l}\text { Ris } \varnothing \text { P2912B SG } \\
\text { Amplifier }\end{array}$ \\
\hline $\begin{array}{l}\text { Tower bottom } \\
\text { bending moment }\end{array}$ & $\begin{array}{l}\text { Micro } \\
\text { Measurement } \\
\text { Strain Gauge } \\
\text { CEA-06-250UW- } \\
350\end{array}$ & Analogue & $\begin{array}{l}\text { Semiconductor } \\
\text { foil subjected to } \\
\text { resistive change } \\
\text { due to bending }\end{array}$ & $\begin{array}{l}\text { Ris } \varnothing \text { P2912B SG } \\
\text { Amplifier }\end{array}$ \\
\hline $\begin{array}{l}\text { Resistor with } \\
\text { gearbox attached } \\
\text { nacelle yaw drive }\end{array}$ & Analogue & $\begin{array}{l}\text { Measuring on } \\
\text { resistive path }\end{array}$ & Voltage divider \\
\hline
\end{tabular}




\subsection{Numerical Simulations}

The methodology highlighted in Sect. 14.2 was applied on the numerical models described in Sect. 14.3.1. The goals of the simulations were to:

- Establish the need for KF based NA tracking

- Establish NA as a damage sensitive feature

- Perform Level II damage detection using Bi-Axial NA tracking

- Establish the robustness of KF based NA tracking methodology

\subsubsection{Need for KF tracking}

The KF as explained in Sect. 14.2.4 combines the previous estimate of the state and the new estimate based on the measurements. Thus in noisy conditions, the KF based estimation is much more stable than the direct estimation. Also, in the second section it was outlined how one can estimate the NA from the instantaneous measured strains. This section compares the NA estimation by these two methods in different simulated conditions. The measured strains and the yaw angle were taken as input to the system and the NA location was estimated by both the methods. The measured signal was contaminated with different levels of white noise in order to simulate real conditions. Table 14.3 shows the statistical performance of the two estimation methods.

As can be seen, the KF needs some initial time for the convergence, but once the convergence is achieved the estimation is more stable. This can be seen from the lower standard deviation in the NA estimate observed while using the KF estimation than that using the direct estimation. Furthermore, it can also be seen that the mean NA position for the KF based estimation is more stable than that using direct estimation. The more stable mean will allow us to set lower thresholds for the change in NA position. This in turn will allow detection of small levels of damage in the tower structure.

Thus, use of KF improves the estimation, and as such, is recommended for accurate NA tracking. Furthermore, the KF algorithm is ideally suited for data

Table 14.3 Statistical performance of estimators (Soman et al. 2015a)

\begin{tabular}{l|l|l}
\hline Scenario & $\begin{array}{l}\text { KF estimation } \\
\text { standard deviation } \\
\text { (mean) }\end{array}$ & $\begin{array}{l}\text { Direct estimation } \\
\text { standard deviation } \\
\text { (mean) }\end{array}$ \\
\hline $0 \%$ Noise for strain and 0\% noise for yaw angle & $0.0444(1.633)$ & $0.1064(1.637)$ \\
\hline $5 \%$ Noise for strain and 0\% noise for yaw angle & $0.0474(1.633)$ & $0.1862(1.622)$ \\
\hline $\begin{array}{l}10 \% \text { Noise for strain and 0\% noise for yaw } \\
\text { angle }\end{array}$ & $0.0507(1.635)$ & $0.4527(1.666)$ \\
\hline $0 \%$ Noise for strain and 5\% noise for yaw angle & $0.0444(1.633)$ & $0.1168(1.641)$ \\
\hline $5 \%$ Noise for strain and 5\% noise for yaw angle & $0.0499(1.633)$ & $0.211(1.686)$ \\
\hline
\end{tabular}


fusion, and hence the yaw angle tracking can be seamlessly included in the algorithm in order to increase the confidence level in the NA tracking.

\subsubsection{NA as a Damage Sensitive Feature}

As explained in the earlier section, the NA of the cross-section of the tower is the property of the condition of the structure and may be used as a damage indicator. In order to validate the use of NA as damage indicator, artificial damage was introduced in one element of the tower, by reducing the flexural rigidity of that particular element by $20 \%$. The simulated damage was detected by comparing the NAE of the damage and the undamaged element. The relative change in the location of the NA is given in Table 14.4.

The damage is detected if the change in the NA estimation of the damaged and undamaged state is more than a specified threshold, which is determined based on simulated studies of different damage scenarios (Soman et al. 2015a). As can be clearly observed, even in the presence of measurement noise, there is significant difference in the change of the NAE of the damage element and the others, so the

Table 14.4 NA based damage detection in presence of noise (Soman et al. 2014a)

\begin{tabular}{l|l|l|l|l}
\hline \multirow{2}{*}{$\begin{array}{l}\text { Sensor } \\
\text { location }(\mathrm{m})\end{array}$} & \multicolumn{5}{l}{ NAE $(\%)$} & $1 \%$ Noise & $5 \%$ Noise & $10 \%$ Noise \\
\cline { 2 - 5 } & $0 \%$ Noise & -0.0694 & -0.2029 & 0.5403 \\
\hline 2.875 & -0.0177 & -0.1266 & -0.3123 & 0.3565 \\
\hline 8.625 & -0.1546 & -0.0576 & -0.1811 & 0.1504 \\
\hline 14.375 & -0.0820 & -0.2161 & -0.3438 & -1.0455 \\
\hline 20.125 & -0.2128 & -0.0508 & -0.0135 & 0.5836 \\
\hline 25.875 & -0.0252 & -0.4577 & -0.4394 & 0.7286 \\
\hline 31.625 & -0.4384 & -1.0899 & -1.0932 & -1.1910 \\
\hline 37.375 & -0.9947 & $\mathbf{5 . 4 6 7 4}$ & $\mathbf{5 . 4 6 7 5}$ & $\mathbf{5 . 3 2 8 6}$ \\
\hline $\mathbf{4 3 . 1 2 5}$ & $\mathbf{5 . 4 8 3 6}$ & -1.0491 & -0.9949 & -0.7893 \\
\hline 48.875 & -1.0367 & -0.5153 & -0.6243 & -0.1339 \\
\hline 54.625 & -0.4416 & 0.0151 & -0.1978 & 0.1797 \\
\hline 60.375 & -0.0422 & -0.2820 & -0.2334 & -0.3370 \\
\hline 66.125 & -0.2413 & -0.0716 & 0.1087 & 0.3750 \\
\hline 71.875 & -0.1271 & -0.1475 & -0.1069 & -0.0487 \\
\hline 77.625 & -0.1664 & -0.0639 & -0.0125 & -0.2715 \\
\hline 83.375 & -0.0417 & -0.1277 & -0.0120 & -0.4831 \\
\hline 89.125 & -0.0770 & 0.0301 & 0.1279 & -0.2785 \\
\hline 94.875 & 0.0101 & -0.0271 & -0.3458 & 0.1291 \\
\hline 100.625 & -0.0121 & 0.0463 & 0.0330 & 0.0299 \\
\hline 106.375 & 0.0584 & 0.0684 & 0.1646 & 0.7457 \\
\hline 112.440 & -0.0368 & & & \\
\hline & & & & \\
\hline
\end{tabular}


chances for a false detection are quite minimal and as such a lower threshold may be possible $1 \%$ in the case where yaw angle is being tracked as well. Thus NA can be used as a robust damage sensitive feature.

\subsubsection{Bi-axial NA tracking}

NA may be used as a damage sensitive feature. Table 14.2 only gives the location of the damage along the altitude. In order to isolate the damage even further, bi-axial NA tracking is necessary. For bi-axial NA tracking, two mutually perpendicular sensor pairs are necessary as explained in Sect. 14.2.3. Then the change in NA can be mapped along the two axes and the exact location determined. In order to show the methodology, four different damage scenarios were simulated, as shown in Fig. 14.3.

Table 14.5 shows the change in NA along the two axes, and as can be seen through decision level data fusion, we can easily isolate the damage accurately.

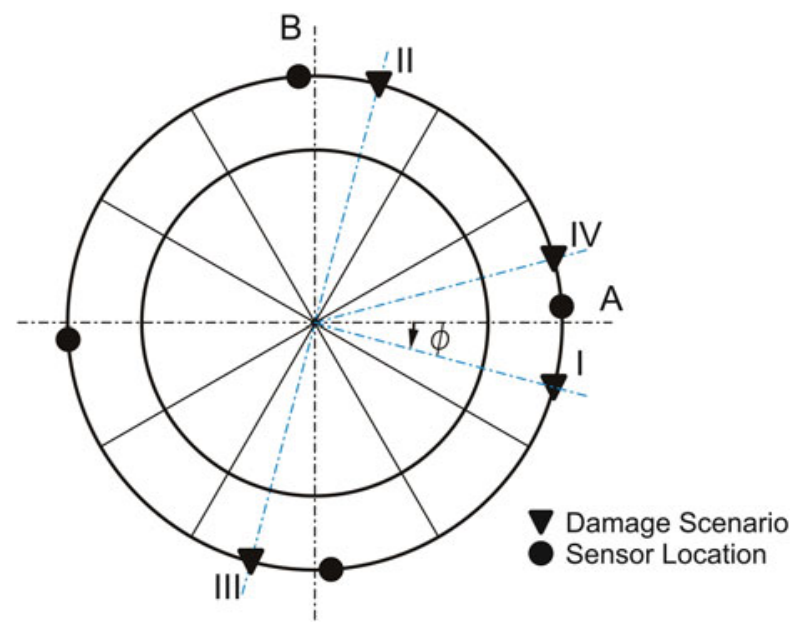

Fig. 14.3 Damage scenarios indicating the need for bi-axial NA tracking (Soman et al. 2015b)

Table 14.5 Bi-axial NA tracking for damage detection (Soman et al. 2015b)

\begin{tabular}{l|l|l|l|l|l|l|l}
\hline & & & & \multicolumn{3}{l}{$\begin{array}{l}\text { Damage location } \\
\text { predicted }\left({ }^{\circ}\right)\end{array}$} & \multicolumn{2}{l}{$\begin{array}{l}\text { Error in detection } \\
\left({ }^{\circ}\right)\end{array}$} \\
\cline { 5 - 9 } $\begin{array}{l}\text { Damage } \\
\text { scenario }\end{array}$ & $\begin{array}{l}\Delta \mathrm{NAE}_{\mathrm{A}} \\
(\%)\end{array}$ & $\begin{array}{l}\Delta \mathrm{NAE}_{\mathrm{B}} \\
(\%)\end{array}$ & $\begin{array}{l}\text { Damage } \\
\text { location }\left({ }^{\circ}\right)\end{array}$ & Fusion & W/o fusion & Fusion & W/o fusion \\
\hline I & 6.264 & -2.020 & -15 & -17.9 & -17.9 & 2.9 & 2.9 \\
\hline II & 2.003 & 6.206 & 75 & 72 & 72 & 3 & 3 \\
\hline III & -2.301 & -6.607 & -105 & -109 & $\mathbf{7 0 . 7}$ & 4 & $-\mathbf{1 7 5 . 7}$ \\
\hline IV & 12.800 & $\mathbf{0 . 8 7 5}$ & 15 & $\mathbf{3 . 8}$ & $\mathbf{3 . 8}$ & $\mathbf{1 1 . 2}$ & $\mathbf{1 1 . 2}$ \\
\hline
\end{tabular}


The damage scenario I and II indicate the validity of the decision level data fusion, and taking the ratio of the changes in NA, is a valid technique for fusion. The damage isolation for case I and II is valid without fusion as the damage lies in the $-90^{\circ}$ to $90^{\circ}$ range. Damage scenario III is a point diametrically opposite to II and it shows the significance of decision level data fusion. If the signs corresponding to the change of the location are not taken into account the isolation give a diametrically opposite point which may be termed as false isolation of the damage (shown in bold values in Table 14.5) and hence the decision level data fusion is necessary. Damage scenario IV indicates that when the damage is at locations perpendicular to one of the axes, the results obtained for the isolation are not accurate, but this in essence proves that bi-axial tracking is indeed necessary.

It can be noted that the change in the location of NA in scenario IV, in the estimate at $\mathrm{A}$, is significant and hence false detection of the damage is highly unlikely. The high change exceeding $10 \%$ also indicates very close proximity of the damage to the strain sensors and gives further idea about the location of the damage. Furthermore, the decision level data fusion will recognize that the threshold for the damage detection has not been exceeded (shown in bold values in Table 14.5) and as such the data should not be used, thus making the decision level data fusion based strategy more robust against false localization.

\subsubsection{Robustness of $\mathrm{KF}$}

The ideal SHM methodology should need limited operator skill and should be objective in nature. The previous section formally introduced a methodology for the selection of the threshold for damage detection which allows the methodology to be objective. In order to make it independent of operator skill the methodology should be robust in nature. This section shows how the KF is a robust methodology requiring limited operator skill for setting up. The Kalman Filter is a powerful tool for the estimation of the state variables especially in the presence of measurement noise. The NA estimate is independent of the initial setting up of the NA, and as such this makes the damage detection methodology robust. Figure 14.4 shows the evolution of the prediction under different parameters of the KF initiation.

Figure 14.4a shows the estimate of NA for different initial guess locations of the NA. As can be seen NA estimate is independent of the starting position, and converges to the true value in relatively short time and as such the damage detection methodology is not affected. Figure 14.4b shows the independence of the initial NA estimate to the uncertainty of the initial guess location or in other words the covariance of the state matrix. The value affects the rate at which the convergence is achieved.

As can be seen lower the value of the covariance quicker the convergence is achieved. Figure 14.4 shows the effect of the uncertainty in the strain measurements on the NA estimate. This uncertainty is termed as measurement noise. 
(a) NA Estimate for different Initial Guess Locations of NA

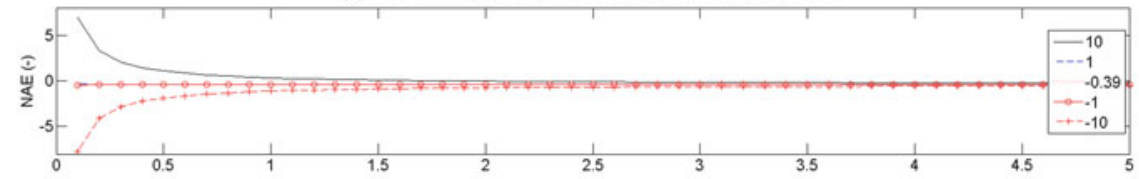

(b) NA Estimate for different confidence levels of initial guess location of NA (covariance of P matrix)

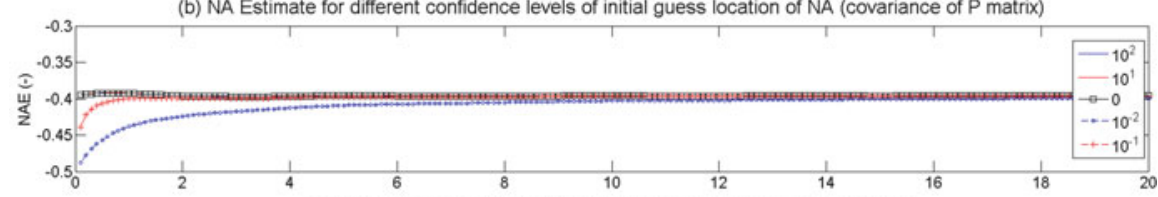

(c) NA Estimate for different uncertainty levels in strain measurement

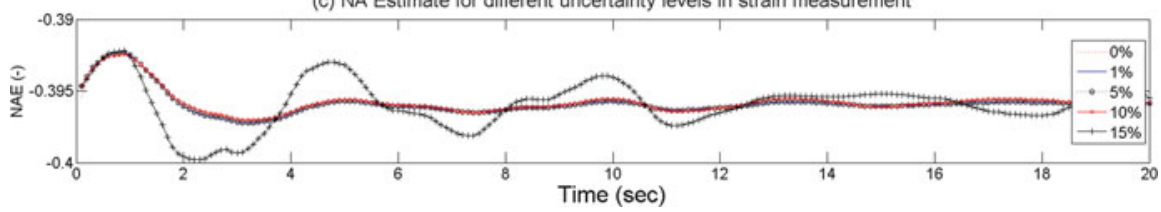

Fig. 14.4 Sensitivity of NAE to KF parameters (Soman et al. 2015a)

It is intuitive to think that greater the measurement noise, more unsteady is the NA estimate, which in turn affects the threshold value for damage detection. It can be seen that even at $10 \%$ measurement noise, the KF estimate is more or less stable, which points towards the robustness of the KF based methodology.

\subsection{Validation}

The success of the methodology for damage detection was checked on simulated damage scenarios. But the methodology needs to be validated with application on real structure. This chapter thus aims at validating the methodology with available strain data from the NTK 500/41 Wind Turbine.

The sensors were placed on the wind turbine as explained in Sect. 14.3, and are capable of measuring only the bending moments. Also, it is not feasible to introduce any damage in the structure, as the structure is in-service. Also the insurance for the machine will be void if such changes are made to the system. Hence, the study is limited to validating the need for KF based NA tracking. The results are shown and discussed in detail in the subsequent sections.

\subsubsection{Need for KF Based NA Tracking}

As explained earlier the direct estimation method for the NA tracking estimates the NA position based on the instantaneous strain measured by the system. In 

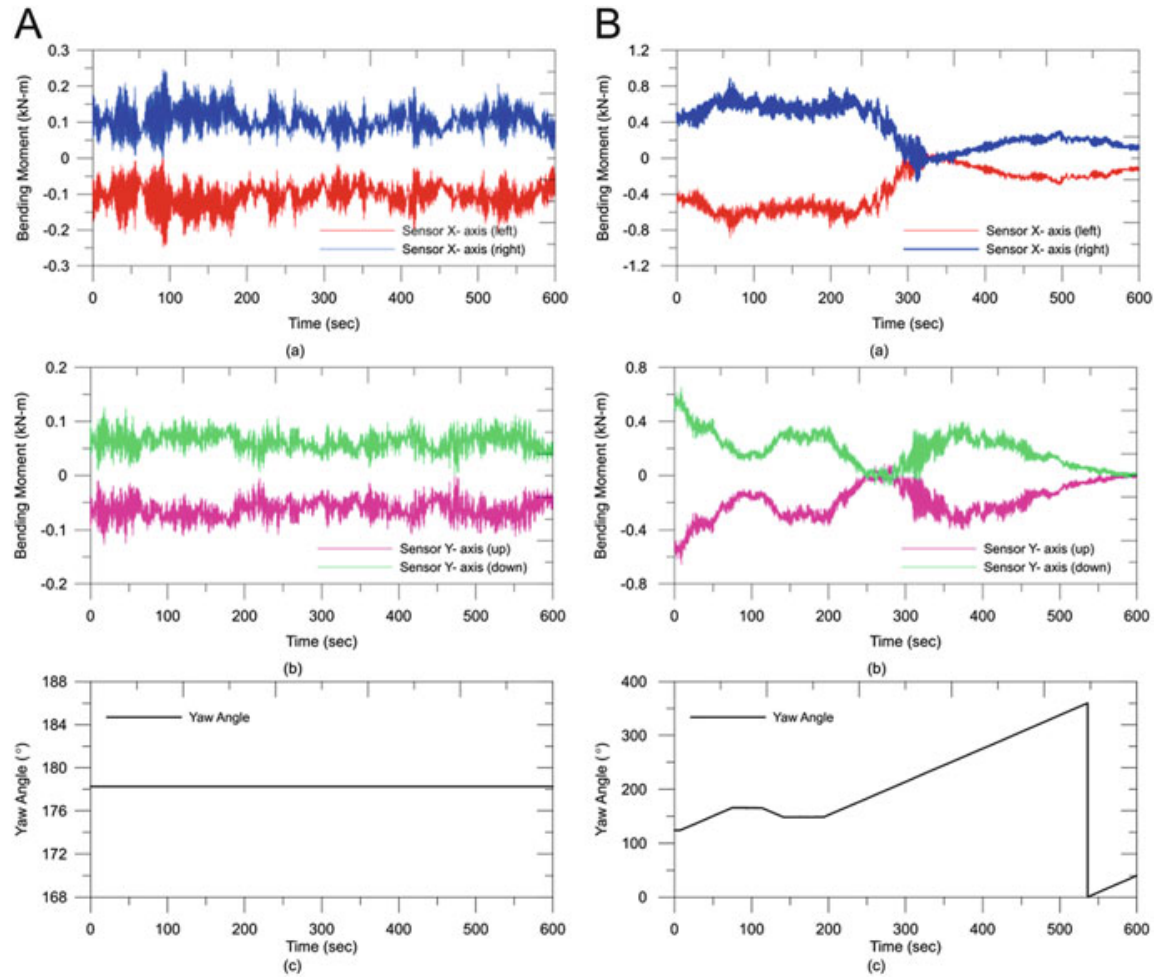

Fig. 14.5 Measurements from Nordtank NTK 500/41 wind turbine. (A) Fixed yaw angle condition, (B) changing yaw angle condition. (a) Bending moment along $\mathrm{x}$-axis, (b) bending moment along $\mathrm{y}$-axis, (c) yaw angle measurement

the presence of measurement noise, this may lead to inaccurate NA estimation. On the other hand KF makes use of some component of the earlier estimate and then incorporates the new measurements of strain, in order to yield a more stable estimation.

In order to ascertain the performance of the two methods, the strain and yaw angle measurements from the Nordtank NTK 500/41 wind turbine were used for the NA estimation. Figure 14.5A(a) and (b) show the strains measured when the yaw angle was more or less constant (Fig. 14.5A(c)). Figure 14.5B(a) and (b) show strain measurements for the condition when the yaw angle was changed constantly (Fig. 14.5B(c)).

Based on these inputs the NA was tracked using the two methods. Figure 14.6 shows the performance of the direct estimation and KF estimation methods for the fixed yaw angle condition and Fig. 14.7 shows the performance in changing yaw angle conditions. The NA estimation is more stable in both the methods for fixed yaw angle conditions as the mass distribution is constant and the bending strains are more or less constant. Also, it can be clearly seen the NAE is more stable using 

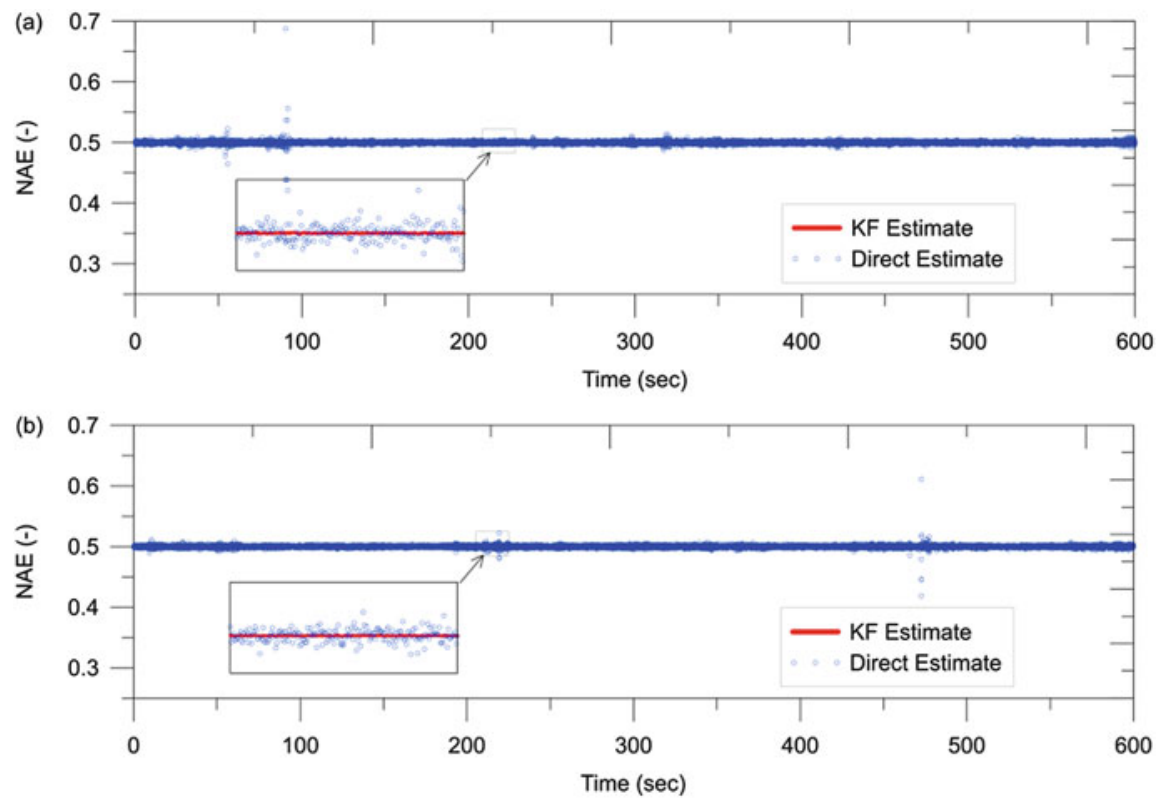

Fig. 14.6 Comparative performance for NA estimation for fixed yaw angle condition (a) along $\mathrm{x}$-axis (b) along $\mathrm{y}$-axis (Soman et al. 2015c)

the KF estimate. The direct method gives erroneous estimation especially when the strain values are close to 0 . This is in keeping with intuition, as when the measured strain is low, the effect of sensor noise and localized changes becomes significant leading to false estimations.

Figure 14.8 gives the comparative performance of the two methods under both the working conditions. It can be clearly observed that the standard deviation of the KF based estimation method is orders of magnitude better than the direct estimation method. Also, the mean of the KF based estimate is more accurate than the direct method.

The more accuracy in the estimation allows us, in turn, to have stricter thresholds for the damage detection. The lower thresholds allow earlier damage detection. Also, in the case of direct estimation, where the standard deviation of the estimate is higher, there is a higher possibility of a false negative damage detection, which leads to higher downtime and lower availability. 

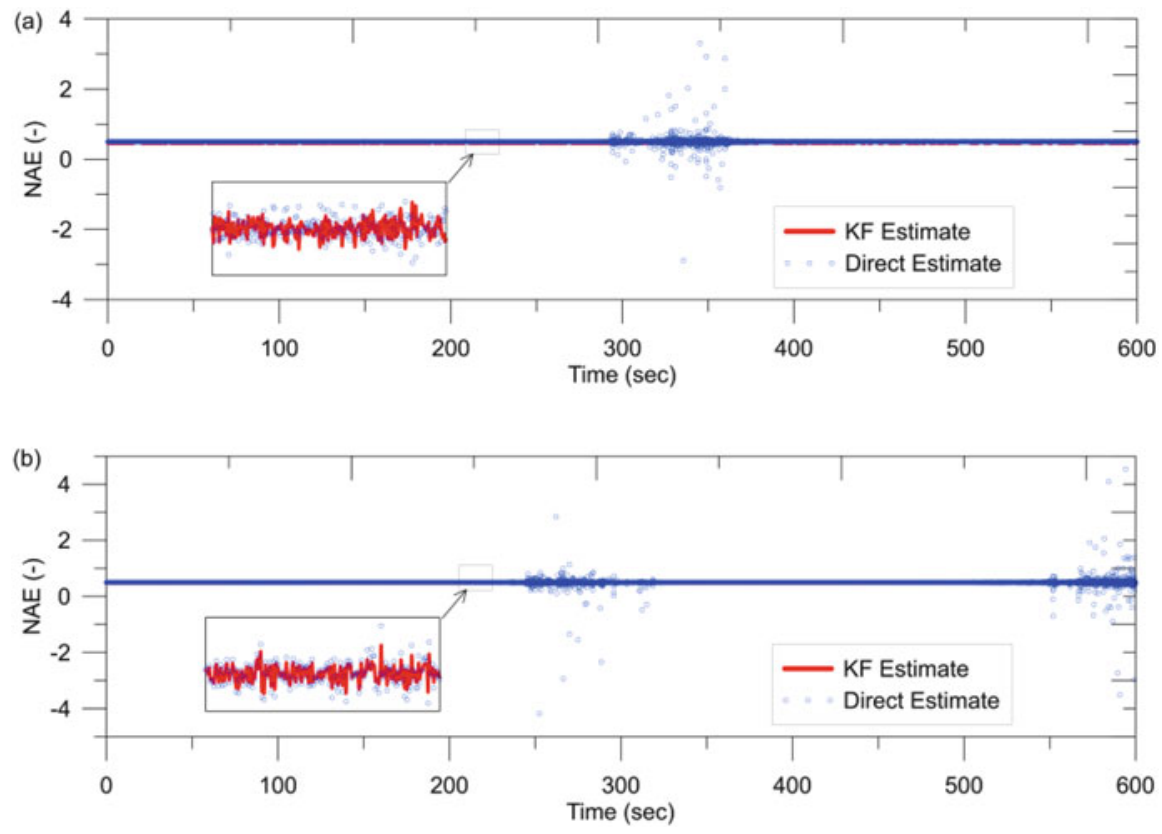

Fig. 14.7 Comparative performance for NA estimation for changing yaw angle condition (a) along $\mathrm{x}$-axis (b) along $\mathrm{y}$-axis (Soman et al. 2015c)

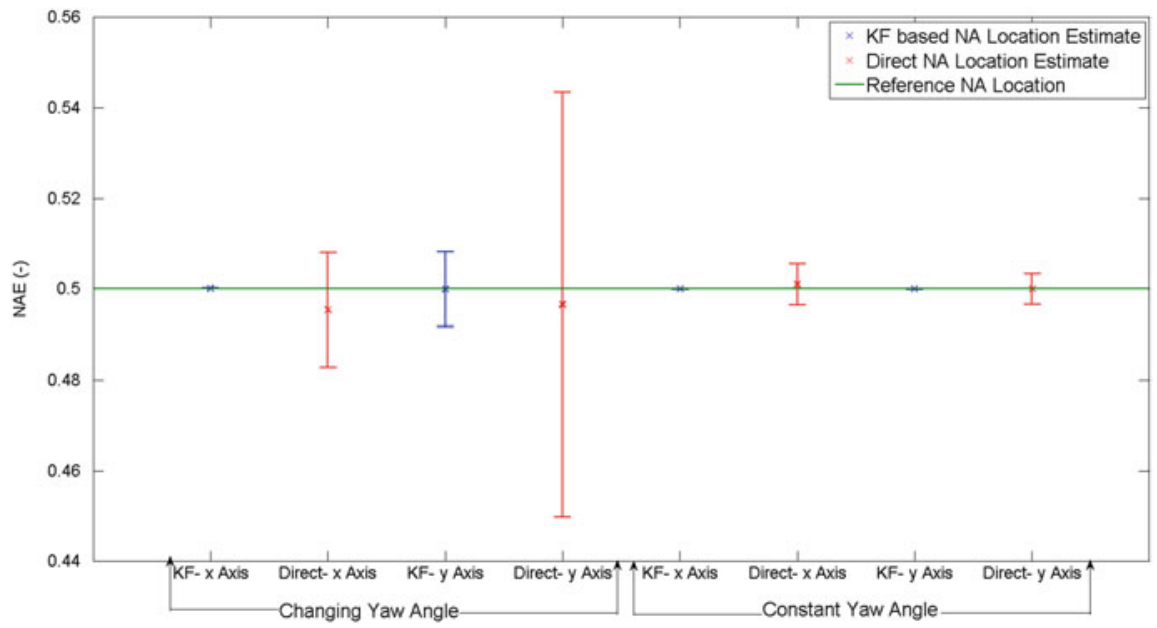

Fig. 14.8 Statistical performance comparison for NA estimation (Soman et al. 2015c) 


\subsection{Conclusions}

The present research presents a complete damage detection methodology for tower structures of offshore wind turbine structures. The methodology makes use of NA as a damage sensitive feature. The NA as a damage sensitive feature has been proposed in literature for bridges (Sigurdardottir and Glisic 2013, 2014), but the innovation of the research is the customization of the NA tracking to the wind energy applications. The method makes use of KF for accurate NA tracking. The KF is robust tool ideally suited for data fusion and allows us to seamlessly combine the yaw angle measurement in the NA estimation and in turn improve the confidence level of the NA estimate. The KF uses the instantaneous strain measurements and a part of the previous estimate of NA location to give a more stable estimate than the one given by the direct method. The KF based estimations are shown to be more precise and accurate as compared to the estimation by the direct estimation. This has been shown through several simulated studies under different conditions of measurement noise as well as through actual strain measurements.

The NA seems to be a robust indicator and is not affected by the ambient loads as well as temperature conditions. Also, through the use of decision level data fusion, one can easily isolate the damage. The method also highlights a method to determine the threshold for the damage detection alarm to be raised. The threshold is chosen based on the trade-off of false detections and this threshold has been successfully implemented on the data from the Nordtank NTK500/41 wind turbine.

In the present study only the bulk temperature effects have been considered, and any temperature gradient effects are expected to be negligible because of the metallic construction and relatively thin cross section. Lastly the blades have been considered to be in braked condition in order to avoid any rotation and the resultant change in mass of the system. It is envisaged that the rotation effects can be easily incorporated through the data fusion of the rotation speed and a wavelet based filtering of the strain cycles due to the rotation. In addition, the present damage detection studies have been limited to reduction in the flexural rigidity of one element of the tower. Although this approach is commonly used in SHM of structures, is not a realistic damage scenario. Thus the performance of the NA tracking methodology must be validated for the detection of some realistic damage scenarios like fatigue cracks, or loosening of the bolts at the connecting flange. These are two areas identified for the future work, along with validation on strain data from a damaged tower structure.

Acknowledgments The authors would like to acknowledge the European Commission for their research grant under the project FP7-PEOPLE-20120ITN 309395 "MARE-WINT" (new Materials and REliability in offshore WINd Turbines technology). The authors would also like to thank DTU Wind Energy, for providing valuable information for the modeling of the 10 MW RWT tower for the purpose of this study. The authors are also grateful to TASK-CI for allowing the use of their computational resources. The opinions expressed in this paper do not necessarily reflect those of the sponsors. 
Open Access This chapter is distributed under the terms of the Creative Commons AttributionNonCommercial 4.0 International License (http://creativecommons.org/licenses/by-nc/4.0/), which permits any noncommercial use, duplication, adaptation, distribution and reproduction in any medium or format, as long as you give appropriate credit to the original author(s) and the source, provide a link to the Creative Commons license and indicate if changes were made.

The images or other third party material in this chapter are included in the work's Creative Commons license, unless indicated otherwise in the credit line; if such material is not included in the work's Creative Commons license and the respective action is not permitted by statutory regulation, users will need to obtain permission from the license holder to duplicate, adapt or reproduce the material.

\section{References}

Adewuyi A, Wu Z, Serker N (2009) Assessment of vibration-based damage identification methods using displacement and distributed strain measurement. Struct Health Monit 8(6):443-461

Bak C, Zahle F, Bitsche R et al (2013) The DTU 10-MW reference wind turbine. In: DTU orbit-the research information system. Available via Technical University of Denmark. http://orbit.dtu.dk/files/55645274/The_DTU_10MW_Reference_Turbine_ Christian_Bak.pdf. Accessed 06 Apr 2016

Bas J, Carriveau R, Cheng S et al (2012) Strain response of a wind turbine tower as a function of nacelle orientation. In: Dini P, Lorenz P (eds) Proceedings of BIONATURE 2012: the 3rd international conference on bioenvironment, biodiversity and renewable energies, St Maarten, 2012

Brown R, Hwang P (1997) Introduction to random signals and applied Kalman filtering. Wiley, New York

Cho $\mathrm{S}$ et al (2010) Structural health monitoring of cable-stayed bridge using acceleration data via wireless smart sensor network. In: Frangopol DM, Sause R, Kusko CS (eds) Bridge maintenance, safety, management and life-cycle optimization: proceedings of the 5th international conference on bridge maintenance, safety and management, Philadelphia, July 2010. CRC Press, London, p 85

Doebling S, Farrar C, Prime M (1998) A summary review of vibration based damage identification techniques. Shock Vib Dig 30(2):91-105

Paulsen U (2011) Verification of long-term load measurement technique: work package 1B.2 under the European Commission, integrated wind turbine design (UPWIND). In: DTU orbit-the research information system. Available via Technical University of Denmark. http://orbit.dtu. dk/files/5712228/ris-r-1782.pdf. Accessed 10 Apr 2016

Sen Z, Altunkaynak A, Erdik T (2012) Wind velocity vertical extrapolation by extended power law. Adv Meteorol. doi:10.1155/2012/178623

Sigurdardottir D, Glisic B (2013) Neutral axis as damage sensitive feature. Smart Mater Struct. doi:10.1088/0964-1726/22/7/075030

Sigurdardottir D, Glisic B (2014) Detecting minute damage in beam-like structures using the neutral axis location. Smart Mater Struct. doi:10.1088/0964-1726/23/12/125042

Soman R, Malinowski P, Ostachowicz W (2014a) Neutral Axis Tracking for damage detection in wind turbine towers. Paper presented at the European Wind Energy Conference and Exhibition (EWEA), Barcelona, 10-13 March 2014

Soman R, Malinowski P, Ostachowicz W (2014b) Kalman Filter based data fusion for neutral axis tracking for damage detection in wind-turbine towers. In: Abstracts of the 7th European workshop on structural health monitoring, EWSHM, Nantes, 8-11 July 2014

Soman R, Malinowski P, Ostachowicz W (2015a) Threshold determination for neutral axis tracking based damage detection in wind turbine towers. Poster presented at the Offshore 2015. European Wind Energy Association, Copenhagen, 10-12 March 2015 
Soman R, Malinowski P, Ostachowicz W (2015b) Bi-axial neutral axis tracking for damage detection in wind-turbine towers. Wind Energy 9(4):639-650

Soman R, Malinowski P, Ostachowicz W et al (2015c) Kalman filter based data fusion for neutral axis tracking in wind turbine towers. In: Kundu T (ed) Proceedings of the SPIE 9438, health monitoring of structural and biological systems, California, 2015

Standards Norway (2009) Eurocode 1: actions on structures-part 1-4: general actions-wind actions. NS-EN 1991-1-4:2005+NA:2009, Lysaker

Welch G, Bishop G (1995) An introduction to the Kalman filter. In: Technical reports, Department of Computer Science University of North Carolina at Chapel Hill. Available via UNC CS. https://www.cs.unc.edu/ welch/media/pdf/kalman_intro.pdf. Accessed 10 Apr 2016

Xia H, Ni Y, Ye X (2012) Neutral-axis position based damage detection of bridge deck using strain measurement: formulation of a Kalman filter estimator. Paper presented at the 6th European workshop on structural health monitoring, Dresden, 3-6 July 2012 